\title{
Low exposure to Plasmodium falciparum and Acquisition of Antibodies to Circumsporozoite Protein Antigens in Individuals Living in Western Highlands of Kenya with Unstable Malaria Transmission
}

\author{
Michael Kisongochi Walukhu ${ }^{\mathrm{a}^{*}}$, Michael M Gicheru ${ }^{\mathrm{b}}$, Robert Wanjala Nyukuric \\ aDepartment of Biological Sciences, Masinde Muliro University of Science and Technology, Kakamega, Kenya. \\ ${ }^{b}$ Department of Zoological Sciences, Kenyatta University, Kenya \\ ${ }^{\mathrm{c}}$ Department of Biological and Environmental Sciences, Kibabii University, Bungoma, Kenya. \\ *Correspondence to Mr. Michael Walukhu (michaelwalukhu@gmail.com)
}

\begin{abstract}
Background: Malaria continues to be a major public health concern despite the concerted efforts to eliminate it. Antibodies to Plasmodium falciparum ( $P$. falciparum) antigens are involved in prevention of infection and disease with some of the antigens being targeted as lead candidates in vaccine development. However, most of the studies have been done in malaria endemic areas with little information available on exposure and acquisition of protective antibodies in areas of low and unstable malaria transmission. This study sought to determine whether the extent of exposure to P. falciparum affect acquisition of protective antibodies by measuring antibody levels and determine the prevalence of circumsporozoite protein (CSP) and crude schizont extract (SE) in individuals living in an area with low unstable malaria transmission in Western Highlands of Kenya.

Methods: Sixty plasma samples from individuals living in an area of low unstable malaria transmission in Western Highlands of Kenya were randomly selected and categorized into three age groups: <8years $(n=25), 8-18 y e a r s ~(n=21)$ and $>$ 18years $(\mathrm{n}=14)$. Antibodies levels in plasma were measured by Enzyme Linked Immunosorbent Assay (ELISA) and compared to known positive and negative control plasma samples. Prevalence and levels of antibodies produced against circumsporozoite protein and schizont extract antigens were compared between age groups.

Results. The prevalence of antibodies ranged from $0 \%$ to $14.29 \%$ at arbitrary units (AU)>2 for the two antigens. The antibody prevalence did not significantly increase with age $(\mathrm{P}>0.05)$ but correlated with CSP and SE antigens ( $\mathrm{r}=0.5977$; $\mathrm{P}<0.05)$.

Conclusion This study highlights antibody responses to CSP and SE antigens in individuals living in low and unstable malaria transmission. The levels of antibodies were generally low across all age groups and there were no significant differences among age groups. Longitudinal study on more antigens is needed to inform exploration of multi-antigen vaccines and also adopt several control measures including Epidemic Preparedness and Response (EPR).
\end{abstract}

Key words: Malaria, Circumsporozoite protein, Schizont extract, ELISA, Plasmodium falciparum, IgG antibodies, Kenya

\section{INTRODUCTION}

$\mathrm{M}$ alaria is a major global health problem and more so in sub-Saharan Africa. It is one of the most important infectious diseases worldwide, causing an estimated

214 million clinical cases and 438,000 deaths annually ${ }^{1}$. Sustainable control of malaria would be enhanced if East Africa Science 2020| Volume 2 | Number 1 effective vaccines were available for use in combination with the available control measures including insecticide treated nets (ITNs), long lasting treated nets (LLTNs), indoor residual spraying (IRS) and epidemic preparedness and response $(\mathrm{EP})^{2}$. Factors that hinders development of malaria vaccine include the complexity in life cycle of 
malaria parasite which involve a succession of stages in the human and the ability of parasite to utilize a number of immune evasion mechanisms ${ }^{3}$.

Malaria is a leading cause of morbidity and mortality in Kenya with about 27 million people ( $70 \%$ of the country's population) at risk of infection ${ }^{4}$. In Kenya, malaria distribution is not uniform due to geographical differences in altitude, rainfall and humidity. These geographical factors influence the transmission patterns as they determine the vector densities and biting intensity where high ambient temperature shortens sporogonic cycle of the mosquito parasite hence shorter duration of the gonotrophic cycle. Four epidemiological zones are determined in Kenya including; endemic areas (with stable malaria transmission in altitudes of $0-1300 \mathrm{M}$ at Kenyan coast and around lake Victoria), seasonal transmission zones (arid and semi-arid), low risk zones (central islands of Kenya including Nairobi) and epidemic zones which covers Western highlands of Kenya with seasonal transmission with considerable year to year variation ${ }^{5}$. The epidemic phenomenon is attributed to increase in minimum temperature during long rains period which favours and sustains vector breeding. The whole population is at risk during epidemics and fatality can be up to ten times greater than in the areas with stable malaria transimission ${ }^{6}$.

Immunoglobulin- $\mathrm{G}(\mathrm{IgG})$ antibodies are produced against a number of malaria vaccine candidate antigens including the pre-erythrocytic antigen $\mathrm{CSP}^{7}$. These antibodies have been associated with some degree of protection from clinical malaria in areas of stable transmission. However, most of information to date is based primarily on observations of naturally infected individuals living in areas of stable malaria transmission with little information in areas of unstable transmission. Residents of such areas could develop partial protection against severe malaria morbidity with increasing age ${ }^{8}$. Children under the age 5 years are at greater risk of infection with $P$. falciparum in malaria holoendemic areas with older children and adults developing partial immunity to malaria. However, this is not the case in areas of low and unstable malaria transmission.

Protective immunity to $P$. falciparum malaria is slowly acquired after several infections and is dependent on the intensity and duration of the individual exposure to the parasite ${ }^{9}$. The target antigen of the protective immunity is the CSP, a major polypeptide covering, which consists of conserved central region of repeating tetramers (B-cell determinants) that is the primary target of humoral response ${ }^{10}$. Indeed, sterile protection has been achieved in experimental settings using high doses of irradiated sporozoites, making the pre-erythrocytic stages of Plasmodium a focus of the malaria vaccine effort ${ }^{11}$. Subunit vaccines based on circumsporozoite protein (CSP), such as RTS, S, are at the center of this endeavor.

Antibodies to Circumsporozoite protein confer some protective immunity in individuals in malaria endemic areas. Although sporozoites are not associated with clinical symptoms, this is a time when parasite numbers in the host are low and their eradication can completely prevent infection $^{12}$. As much as the protective mechanisms have not been completely fully understood, they are postulated to partly involve humoral immune responses elicited by preerythrocytic antigens as a result of repeated sporozoite infection ${ }^{13}$. It has also been suggested that $\operatorname{IgG}$ and its subclass antibodies to CSP could be important surrogate markers of resistance to infection and reduced malaria morbidity in endemic areas of Africa ${ }^{14}$.

Previous studies have determined that antibodies to schizont extract and CSP occur frequently in individuals living in malaria endemic areas ${ }^{15}$. The antibody levels and prevalence are also proposed to be indicators of malaria exposure and transmission ${ }^{16}$. Most of the studies have been done in areas of high and stable malaria transmission and little done in areas of low and unstable malaria transmission. The present study aimed to assess age dependent immune response against SE and CSP among individuals living in an area with low unstable malaria transmission.

\section{METHODS}

\section{Study site}

The study was conducted in a highland area of Kamasai in the western highlands, Kakamega County, bordering Nandi County, Kenya, where malaria transmission is low, unstable and seasonal. The site is located at an altitude of between 1734 and $1822 \mathrm{M}$ with an approximate population of 3000 as of 2009 census. There are usually two rainy seasons with short season in October and long rains between March and May. Malaria transmission at the site is low and unstable during long rains. It is usually episodic with local outbreaks of malaria with high rates of morbidity and mortality having occurred in the past ${ }^{17}$. Both $P$. falciparum infection and Plasmodium malariae infection have been documented in the $\operatorname{area}^{18}$.

\section{Study population}

The study was carried out in the month of May when transmission was expected to be at peak. Study participants were males and females of all ages who had not moved out of the study area for at least six months by the start of the study and who were residents of the area during the study. This was to minimize the confounding effects of travel and acquisition of infection in nearby lowland areas where malaria is endemic. The enrolment of the participants was 
done by study assistants recruited from the locals and signs and symptoms recorded by a trained clinician.

\section{Inclusion and exclusion criteria}

The participants included in the study were male and female age not limiting, residents of the area who had not moved out of the study site six months prior to commencement of the study and were willing to take part in the study by signing the written consent. Individuals who had haemoglobin values below $8 \mathrm{gHb} / \mathrm{dL}$ and were evidently ill according to the assessment of the clinician were excluded from the study. Also those who declined or whose parents/guardians declined to sign the written consent were excluded.

\section{Sample size determination}

Sample size was determined using StatsToDo online statistical software for estimating correlation coefficient (www.statstodo.com). With the following parameters:

Probability of type 1 error $(\alpha)=0.05$

Expected correlation coefficient $(\rho)=0.32$

At power $(1-\beta)=0.8(80 \%)$

This gave a sample size of 59

The assumption was that approximately $5 \%$ declined to give consent. Since the study involved a one-time point sample collection and only those who had not moved out of the study site for at least six months were eligible, migration after sample collection would have no effect on the results. The number was adjusted to 60 participants in the study site.

\section{Study Design and Data Collection}

The study was cross-sectional and was part of a larger study which involved one time point collection of blood and plasma samples. Prior to sample collection, signs and symptoms of malaria were recorded. Thick and thin smear microscopy was used to diagnosis malaria. Haemoglobin levels were measured using photometry (HaemoContol EKF Diagnostics, and Barleben, Germany). Sixty plasma samples $(n=60)$ were randomly selected from the samples of the larger study and used in the present study. The samples were categorized into three (3) groups based on the age; <8years $(n=25), 18$ years $(n=21)$ and $>18$ years $(n=14)$ to assess the effect of age on $\mathrm{IgG}$ responses. Seven plasma samples $(\mathrm{n}=7)$ from North American individuals who were never exposed to malaria were used as negative controls. Thirty (30) pooled plasma samples from adults living at Asembo Bay in Nyanza, an area of stable malaria transmission was used as positive control. Measurement of IgG responses to crude schizont extract (SE) and circumsporozoite protein (CSP) was done by Enzyme Linked Immunosorbent Assay (ELISA).

\section{Ethical consideration}

Since this study involved both adults and minors, written informed consent was obtained from the study participants or, in the case of minors, from their parents or guardians. The consent forms were signed by participants or their parents/guardians before witnesses. The forms were translated into the local language, Kalenjin (Nandi) for participants and parents/guardians who could not understand English. The samples from the participants were assigned arbitrary numbers to conceal the identity of the participants. Study participants who tested positive for malaria were treated for free at the Health Centre using the recommended line of malaria treatment. Ethical review and approval for this study was obtained from Kenyatta University Ethical Review committee and Ethical Review Committee of Kenya Medical Research Institute.

\section{Laboratory Procedures}

The presence of antibodies to CSP was tested by using recombinant central repeat sequence peptides of CSP consisting of five Asparagine-Alanine-Asparagine-Proline $(\mathrm{NANP})_{5}$ repeat tetrapeptide to which individuals from areas where malaria is endemic demonstrate $\mathrm{IgG}$ responses. Antigens for schizont extract (SE) were obtained from malaria culture using $P$. falciparum from 3D7 parasite isolates ${ }^{19}$. We measured Immunoglobulin G (IgG) antibodies by enzyme-linked immunosorbent assay (ELISA). ${ }^{20}$ We coated separate microtitre plates (NuncImmuno ${ }^{\text {TM}}$ Microwell ${ }^{\text {TM}} 96$ well solid, SIGMA) with $50 \mu 1$ of Circumsporozoite peptide and crude schizont extract separately dissolved in $1 \mathrm{x}$ tris-buffered saline (TBS) to a concentration of $10 \mu \mathrm{g} / \mathrm{ml}$. Following overnight incubation at $4{ }^{\circ} \mathrm{C}$, we washed the plates $4 \times$ with TBS $0.05 \%$ Tween 20 using an automated plate washer and blocked for nonspecific binding with $5 \%$ (wt./vol) bovine serum albumin (BSA) in TBS. We added $50 \mu 1$ of plasma samples diluted to $1: 100$ in $5 \%$ BSA in TBS to wells in duplicates and incubated for 2 hours at room temperature. After washing with washing buffer, we added $50 \mu 1$ of alkaline phosphatase-conjugated goat anti-human IgG (Jackson ImmunoResearch, West Grove, PA) diluted $1: 1,000$ in $5 \%$ BSA in TBS and incubated for 1 hour. After extensive washing (6x) with washing buffer we added $\mathrm{p}$ nitrophenyl phosphate substrate as per the manufacturer's instructions (Sigma, S0942 St. Louis, MO). After colour development we measured the optical density (OD) at $405 \mathrm{~nm}$ on a 96-well ELISA plate reader (Molecular Devices, Sunnyvale, CA). We tested all samples in duplicates. We used pooled plasma from 30 individuals from malaria endemic Lake Victoria region as positive control on each plate to achieve Standardization of the plates. We determined the background from the wells with no plasma (blanks). We deducted the background from the mean OD of each sample and a cut-off threshold of positivity was determined as the mean plus 3 standard deviations from the seven negative control plasma samples 
(Canadians) which we included in each assay. We expressed the IgG antibody levels in arbitrary units (AU). We calculated the numbers of AU by dividing the optical density of a sample by the mean optical density plus 3 standard deviations (SD) for plasma from seven Canadians who had never been exposed to malaria. We determined two threshholds; AU> 1 for low level antibody responses and AU $>2$ for high level antibody responses.

\section{Data Analysis}

Microsoft Office Excel 2007 was used in the initial processing and management of data and further analysis was done using GraphPad Prism 6 statistical software (Prism v6.0, GraphPad Software Inc., La Jolla CA). Wilcoxon rank sum test was used to determine summary statistics for the age groups. Kruskall-Wallis test was used to assess differences in the antibody responses in the various age groups. The non-parametric tests were used since the IgG response data was not normally distributed. The spearman and the proportion of IgG-positive individuals to CSP and $\mathrm{SE}(\mathrm{P}=0.05)$. When the cut-off was set at $\mathrm{AU}>2$ (high level antibodies), the proportion of positive individuals dropped to $4(6.7 \%$ ) for both SE and CSP (Table 1). The total IgG levels to schizont extract (SE) ranged from 0.140-6.260 AU with median level of $0.575 \mathrm{AU}$ and mean of $0.8602 \mathrm{AU}$ in rank correlation test was used to analyze the relationship between IgG responses to SE and CSP. Results were considered significant at $P<0.05$.

\section{RESULTS}

Microscopy results from peripheral blood smears of participants at the time of sampling demonstrated that the individuals were non-parasitemic and asymptomatic. Measurement of haemoglobin (HB) levels revealed that $53(88.3 \%)$ of participants had HB levels above the cut-off of anaemia at their various age groups. The proportion of antibody-positive individuals at a cut-off of AU> 1 (low level antibodies) was relatively low for both Schizont extract (SE) and circumsporozoite protein (CSP). The results showed that $11(18.3 \%)$ were $\mathrm{IgG}$ positive for SE while $12(20.0 \%)$ were IgG-positive for CSP. There was no difference in proportion of IgG-positive individuals to CSP

the 60 individuals of the study population. On the other hand, the total IgG to circumsporozoite protein ranged from 0.020 4.22 AU and the median level was $0.530 \mathrm{AU}$ and mean of $0.8075 \mathrm{AU}$ in the 60 individuals (Table 2). Figure 1 depict correlation between IgG levels to SE and CSP in the study sample $(\mathrm{r}=0.5977 ; \mathrm{P}<0.001)$.

TABLE 1. Proportion of antibody-positive individuals to Plasmodium falciparum Schizont extract and Circumsporozoite protein at a cut-off of $A U>1$ and $A U>2$

\begin{tabular}{cccccc}
\hline Antigen & \multirow{2}{*}{$\begin{array}{c}\text { Number } \\
(\mathbf{N})\end{array}$} & \multicolumn{2}{c}{ IgG Positive at AU>1 } & \multicolumn{2}{c}{ IgG Positive at AU>2 } \\
\cline { 3 - 6 } & & Number (n) & Percentage (\%) & Number (n) & ${\text { Percentage }(\%)^{*}}^{*}$ \\
& 60 & 11 & 18.3 & 4 & 6.7 \\
SE & 60 & 12 & 20 & 4 & 6.7 \\
\hline
\end{tabular}

TABLE 2. Study Participants' Median and Mean IgG levels (AU) to Plasmodium falciparum Antigens

\begin{tabular}{lcc}
\hline Parameter & Antibody response to SE (AU) & Antibody response to CSP (AU) \\
\hline Number of participants (N) & 60 & 60 \\
Minimum & 0.1400 & 0.0200 \\
$\mathbf{2 5 \%}$ percentile & 0.4300 & 0.3475 \\
Median & 0.5750 & 0.5300 \\
$\mathbf{7 5 \%}$ percentile & 0.7775 & 0.9075 \\
Maximum & 6.2600 & 4.2200 \\
Mean & 0.8602 & 0.8075 \\
Std. deviation & 1.0492 & 0.8110 \\
Std. error of mean & 0.1354 & 0.1047 \\
\hline
\end{tabular}


FIGURE 1. Correlation of antibody levels between schizont extracts (SE) and Circumsporozoite protein (CSP) for all participants $(n=60)$ in an area of unstable malaria transmission.

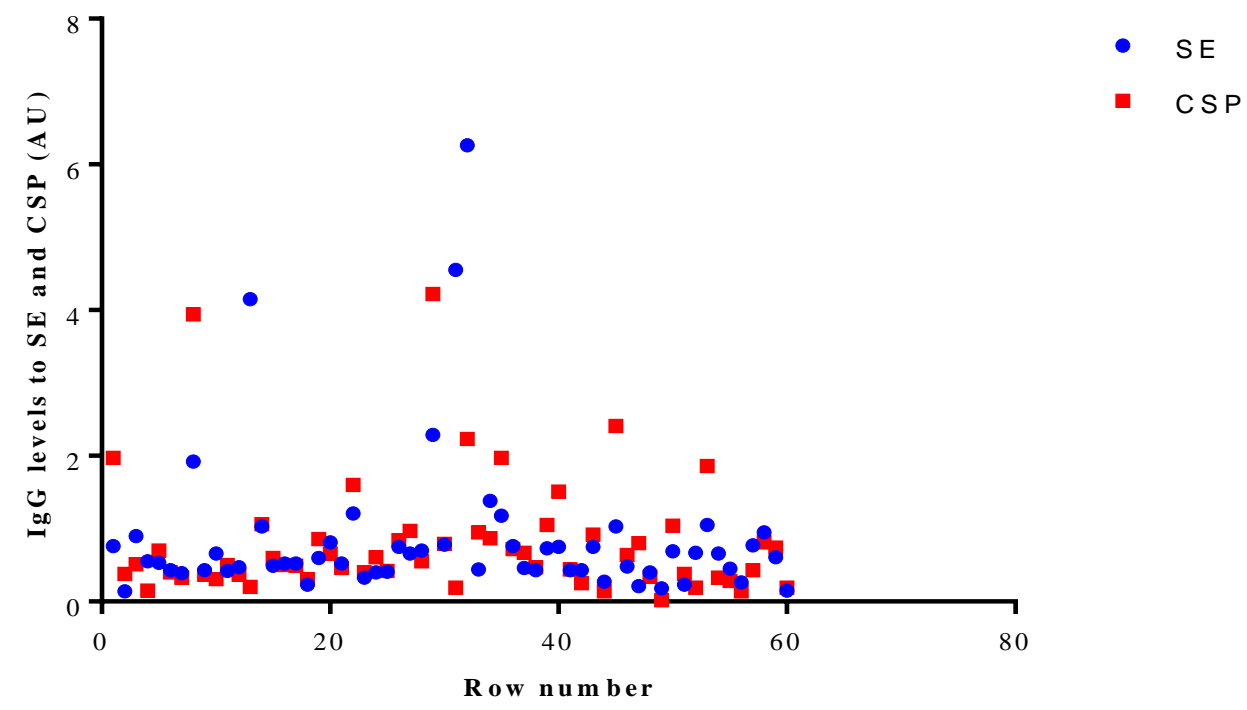

IgG levels were expressed as optical density (OD) in arbitrary units (AU). Correlation coefficient $(r=0.5977), 95 \%$ confidence interval $(0.3988$ to 0.7428$)$ and two-tailed $P$ value of $<0.001$.

Analysis of frequency of IgG to $P$. falciparum by age group is depicted in (Figure 2). When antibody-positive cut off was set at AU>1 (low level antibodies), 2 (8.0\%) of participants aged $<8$ years, $5(23.8 \%)$ of participants aged 8 18 years and $4(28.6 \%)$ of participants above 18 years were IgG-positive to SE. the respective proportion of IgG-positive participants to CSP at cut-off of AU>1 was 4(16\%), $4(19.04 \%)$ and $4(28.6 \%)$ for participants of age groups $<8$ years, $8-18$ years and $>18$ years. The antibody frequency for individuals aged $>18$ years was the same for both SE and CSP (Figure 2).

When the threshold for antibody positivity was considered at $\mathrm{AU}>2$ (High-level antibodies) the proportion of individuals with positive antibody dropped to $6.7 \%$ for both SE and CSP. No antibody against SE and CSP was detected in younger age group when the cut-off point was set at $A U>2$. Interestingly, at cut-off of $A U>2$ the frequency of IgG to CSP was much lower in the individuals aged above 18 years $(7.14 \%)$ than in individuals aged 8-18 years $(14.29 \%)$ though not significantly different $(p>0.05)$.

Similarly, the IgG frequency in age group $>18$ years at cutoff of AU>2 was lower to CSP (7.14\%) than to SE (14.29\%). However, the difference was not significant $(\mathrm{P}>0.05)$ (Figure 3). There was no significant association between median IgG levels and age (Table 3) When the median levels of IgG antibodies to SE were compared to CSP among the three age groups, the levels to CSP were slightly higher than $\mathrm{IgG}$ levels to SE. However, the difference was not significant $(\mathrm{P}>0.05)$ (Figure 4). 
FIGURE 2. Prevalence of total IgG antibodies, determined by ELISA for P. falciparum Schizont extract (SE) and Cicumsporozoite Protein (CSP) in participants of different age groups in an area of unstable malaria transmission.

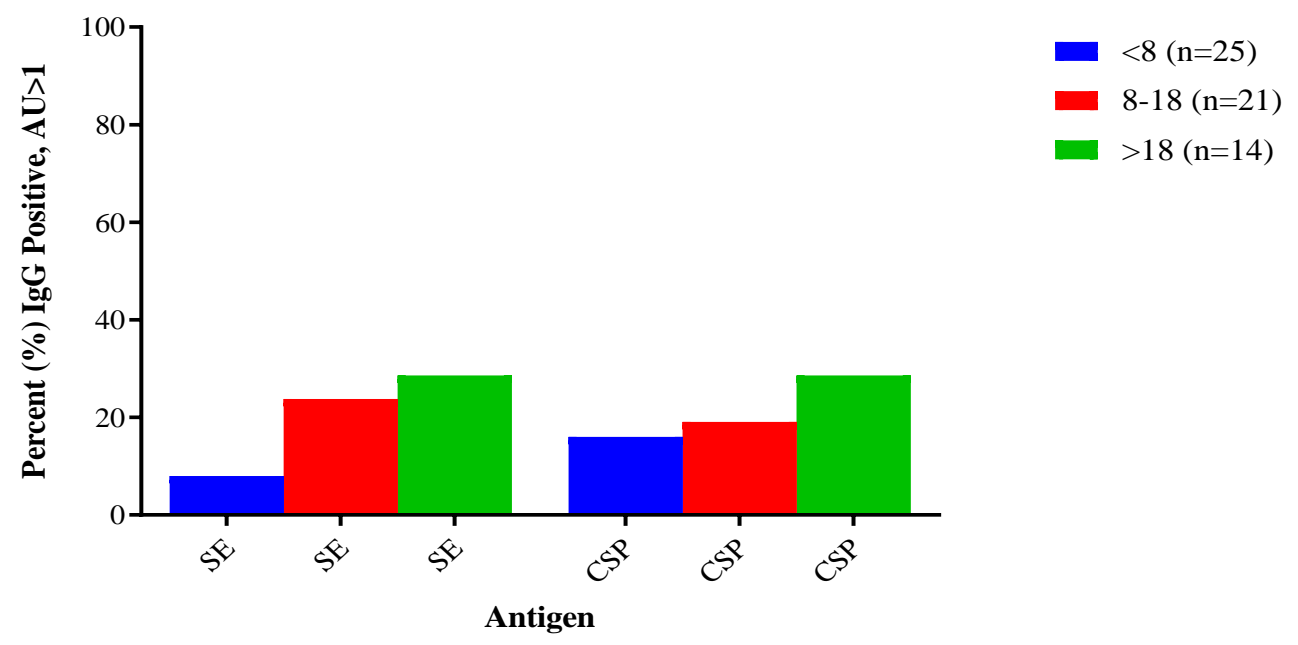

The frequencies were expressed in percentage (\%) when the cut-off of AU>1 was considered antibody-positive (low-level antibodies)

TABLE 3. Median Levels of IgG Antibodies to Plasmodium falciparum Schizont Extract and Circumsporozoite Protein by age groups $A U>1$

\begin{tabular}{ccccc}
\hline Antigen & Age (Years) & $\begin{array}{c}\text { Number of } \\
\text { Participants (N) }\end{array}$ & Median level (Range) & 95\% CI of Median \\
\hline SE & $8-18$ & 25 & $0.5500(0.1400-1.050)$ & $(0.3721-0.6140)$ \\
& $>18$ & 21 & $0.6000(0.2300-4.150)$ & $(0.4700-0.7800)$ \\
CSP & 14 & 25 & $0.6050(0.2700-6.260)$ & $(0.4300-1.3800)$ \\
& $8-18$ & 21 & $0.3800(0.0200-1.970)$ & $(0.4600-0.90 .7000)$ \\
& $>18$ & 14 & $0.6000(0.2000-4.220)$ & $(0.2500-1.5100)$ \\
\hline
\end{tabular}


FIGURE 3. Prevalence of total IgG antibodies, determined by ELISA for P. falciparum Schizont extract (SE) and Cicumsporozoite Protein (CSP) in participants of different age groups in an area of unstable malaria transmission.

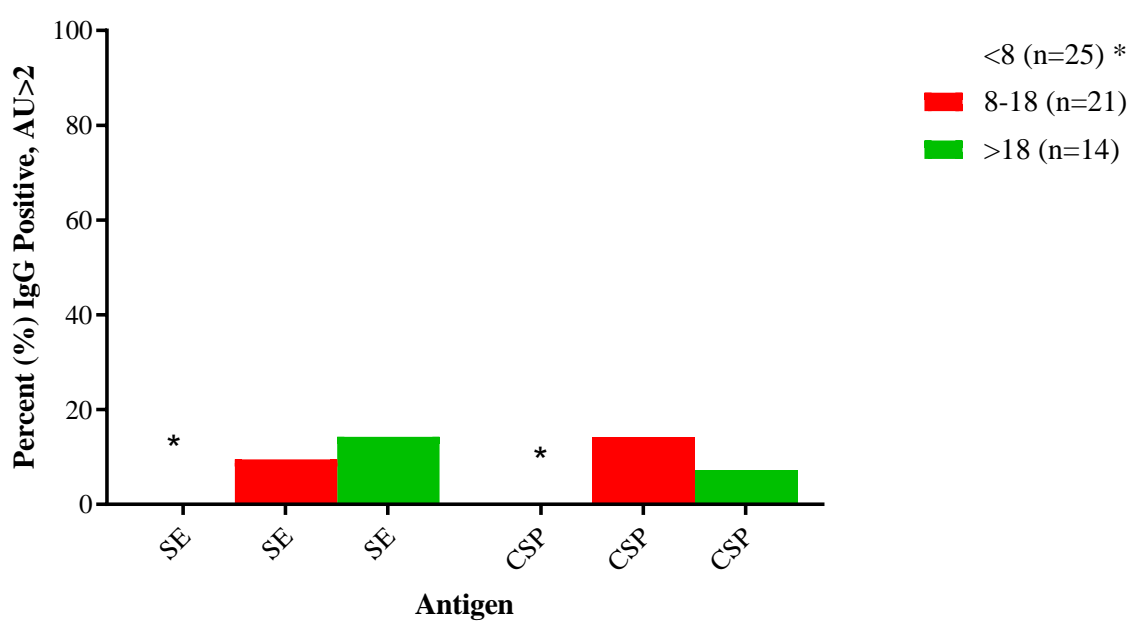

The frequencies were expressed in percentage $(\%)$ when the cut-off of $>2 \mathrm{AU}$ was considered antibody-positive (high-level antibodies). $*=0 \%$ prevalence of IgG antibodies.

FIGURE 4. Relationship between IgG levels to schizont extract (SE) and circumsporozoite protein (CSP) among participants of different age groups in an area of unstable malaria transmission.

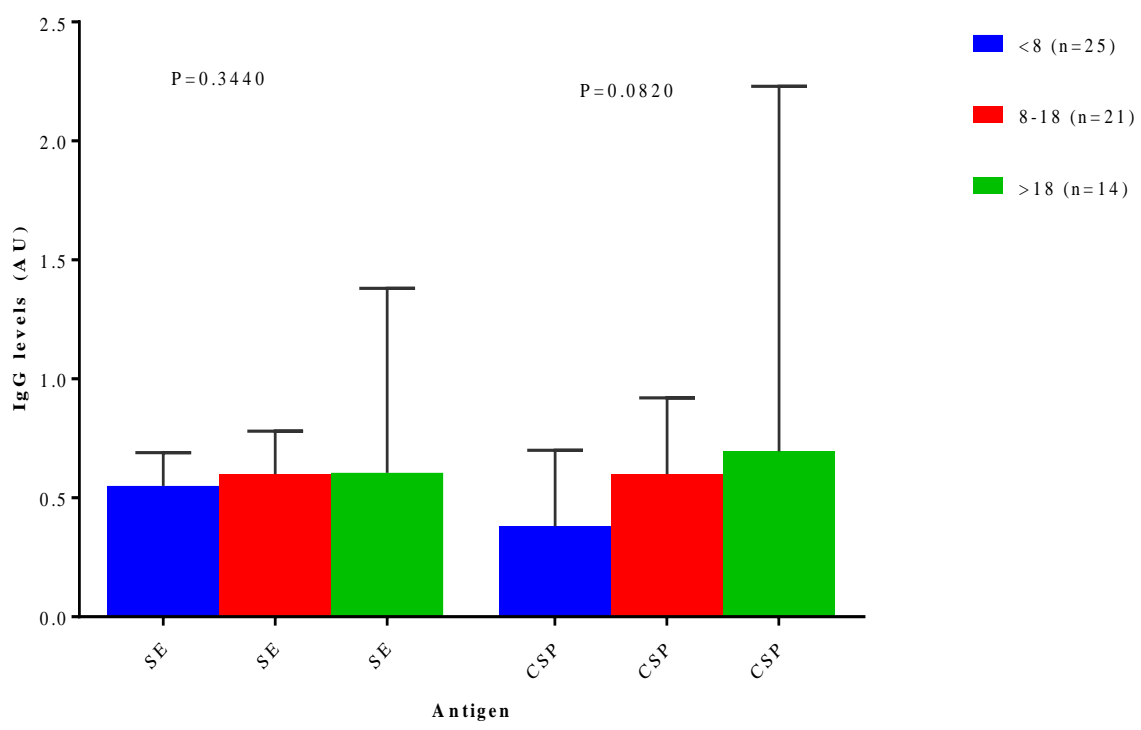

IgG levels were expressed as optical density (OD) in arbitrary units (AU), error bars represent standard error of the mean (SEM). There was no significant difference when medians were compared by Kruskal-Wallis Test $(\mathbf{P}>\mathbf{0 . 0 5})$. 


\section{DISCUSSION}

Previous studies have documented that the presence of highlevel IgG antibodies to pre-erythrocytic antigens especially to CSP correlates with protection from P. falciparum infection in adults and clinical malaria in children ${ }^{7}$. Data from the present study show that generally a very small proportion of individuals living in the study area had highlevel IgG antibodies to the two pre-erythrocytic $P$. falciparum antigens, CSP and schizont extract. This percentage is relatively higher than $3.3 \%$ documented in an earlier study in the same area ${ }^{21}$. This difference may be partly due to timing in sample collection. All the same, the results of the present study demonstrate that the prevalence of high-level IgG antibodies in an area with unstable malaria transmission is extremely low as compared to documented results $(44.3 \%)$ for an area of stable malaria transmission ${ }^{21}$. These findings suggest in part that the intermittent risk of clinical malaria in individuals living in an area of unstable malaria transmission may relate to the low or absence of high-level IgG antibodies to pre-erythrocytic antigens particularly CSP. Low levels of antibodies to crude schizont extract indicate low exposure to $P$. falciparum. As much as presence of high-level IgG antibodies to pre-erythrocytic antigens may not completely prevent blood-stage infection, it may control the parasite density and the clonality of parasitemia by reducing the invasion of $P$. falciparum sporozoites into the liver or by impairing progressive development of parasites in the liver. This notion is supported by documented results in earlier studies that, an increased level of anti-CSP antibodies is associated with decreasing parasite densities in asymptomatic adults in malaria endemic areas ${ }^{22}$. Reduced parasitemia density may also reduce haemolysis of erythrocytes thereby resulting in high haemoglobin levels. Findings in the present study document reduced prevalence of anti-CSP and anti-SE antibodies. This may in part explain the absence of protective immunity among individuals in the study area resulting in frequent epidemics.

The present study generally reports very low IgG antibodies levels to both schizont extract and circumspozoite protein in highland area with low unstable malaria transmission. The levels compared to documented data from areas of high transmission are extremely low. Documented information associates high levels of antibodies to CSP and other preerythrocytic antigens such as MSP, LSA and TRAP with protection against clinical malaria ${ }^{20}$. The low levels $\mathrm{IgG}$ antibodies to CSP and SE reported in the present study suggest in part why the highland areas are prone to epidemics attributed to low exposure to malaria parasite. The findings in the present study that there is a correlation between IgG antibodies levels to the two antigens conform to documented data of strong correlation in expression of antibodies to pre-erythtrocytic antigens especially CSP,
MSP, LSA and TRAP in malaria holoendemic areas ${ }^{20}$. The results in the present study suggest that protection against malaria may be as a result of antibodies to pre-erythrocytic antigens and that repeated exposure and infection appears to be the major determinant of high levels of $\mathrm{IgG}$ antibodies to these antigens. The mechanism of association of antibodies to different pre-erythrocytic antigens in protection is not clearly understood. However, one potential mechanism for antibody related protection is the combined effects of antibodies that target different steps during the preerythrocytic phase of infection life cycle.

Circumsporozoite protein is important in adhesion of the sporozoite to the basolateral membrane of the hepatocyte ${ }^{23}$ and monoclonal antibodies to CSP inhibit parasite invasion of hepatocytes ${ }^{24}$. Thrombospondin related adhesive protein (TRAP) is essential for sporozoite gliding motility ${ }^{25}$ and hepatocyte invasion and antibodies to TRAP have also been shown to prevent sporozoite invasion of hepatocytes ${ }^{26}$. The low levels of IgG antibodies to pre-erythrocytic antigens particularly CSP in highland areas of Western Kenya therefore is attributed to infrequent exposure to malaria parasites hence lack of protective immunity. This low and infrequent exposure is evidenced by low levels of antibodies to schizont extracts in the present study. The degree of natural exposure to malaria required to attain high levels of IgG antibodies to pre-erythrocytic antigens is not known. These high levels of IgG are usually attained by some children in malaria holoendemic areas thereby developing immunity but not others. However, findings of the present study present very low levels of anti-CSP and anti-SE IgG antibodies which may in part be attributed to very low degree of natural exposure to malaria.

In highland areas of Africa, older children and adult continue to be at risk for clinical malaria, although the risk is somewhat lower than that for young children ${ }^{27}$. This is in contrast with areas with stable, high level malaria transmission, where clinical immunity to malaria develops by the age of 3 to 5 , with intermittent episodes of mild malaria and very few episodes of malaria occurring after this age $^{28}$. Results from the present study demonstrate very low prevalence of high-level antibodies to both CSP and schizont extract in individuals of all ages. These frequencies are generally low in comparison to results documented for studies done in areas of stable malaria transmission where over $50 \%$ individuals show high level IgG antibodies to preerythrocytic antigens ${ }^{20}$.

Several previous studies have demonstrated that high levels of IgG antibodies to multiple pre-erythrocytic antigens are associated with protection against $P$. falciparum infection in adults living in malaria-holoendemic area ${ }^{7}$. Majority of this protection is associated with high-level IgG antibodies to 
CSP and LSA not assessed in the present study ${ }^{20}$. The findings of the present study suggest that the persistent risk of clinical malaria in older children and adults in areas of unstable transmission may be as a result of low prevalence of high-level IgG antibodies to pre-erythrocytic antibodies especially CSP.

The immunity to malaria that develops with age in areas of stable malaria transmission is not completely characterized. However, several studies have documented a correlation with protection from clinical malaria for anti-CSP IgG antibodies assessed in this study and IgG antibodies to other antigens not tested in the present study such as LSA-1, TRAP, AMA-1, MSP-1, MSP-2, MSP-3 and GLURP $^{7,29,30,31,32}$. These previous studies confirm the association of age dependent acquisition of anti-malaria antibodies with cumulative exposure to malaria in areas with high and stable malaria transmission. However, little comparable information is available for individuals residing in areas of unstable, epidemic-prone malaria transmission. A study conducted in Tanzania dwelt majorly on blood-stage antigens including MSP-1 19 MSP-2, AMA-1 and glycosylphosphatidylinositol (GPI) across a wide range of ages and transmission intensities. The Tanzanian study reported that antibody isotypes for the tested antigens relate to age and transmission intensity ${ }^{33}$. The present study dwelt on pre-erythrocytic antigens which the Tanzanian study did not test. The key finding of the present study is that highland areas of western Kenya with unstable malaria transmission present extremely low levels of $\mathrm{IgG}$ antibodies as compared to documented data of areas of stable malaria transmission. Furthermore, the results of this study indicate that there is no significant difference in IgG antibody levels to CSP and schizont extract in the study area. Although results of the present study indicate that the levels of $\mathrm{IgG}$ antibodies to CSP and schizont extract increase with age, adults have very low levels of anti-CSP and anti-SE IgG antibodies suggesting why adults are also at risk of malaria in areas of unstable malaria transmission. The association of $\mathrm{IgG}$ antibodies to CSP tested in this study with protection from $P$. falciparum and disease and the striking low or absence of high-level antibodies in the study area, suggests that these responses may be important in protection against infection and disease. The idea that immune response to CSP is important for development of clinical immunity against malaria is supported by the success of CSP-based RTS, S vaccine in inducing protection from clinical malaria due to $P$. falciparum in children. Indeed, the first results of phase 3 trial which was launched in 2009 in seven African malariaendemic countries; Gabon, Ghana, Kenya, Malawi, Burkina Faso, Tanzania and Mozambique showed that RTS,S reduced the risk of children experiencing clinical malaria and severe malaria by $39 \%$ and $29 \%$ respectively ${ }^{34}$. The vaccine is now undergoing pilot implantation in 3 to 5 settings in sub-Saharan Africa ${ }^{35}$. Therefore, the low levels of IgG antibodies to CSP and schizont extract in the area of low and unstable malaria transmission reported in the present study could be part of the reason for impaired clinical immunity in adults resulting in epidemics.

\section{CONCLUSION}

This study highlights the antibody responses to CSP and SE antigens in individuals living low and unstable malaria transmission. The levels and prevalence of $\mathrm{IgG}$ antibodies response to SE and CSP were generally low across all age groups and there were no significant differences among age groups. Longitudinal study on more antigens is needed to inform exploration of multi-antigen vaccines and other malaria control measures including Epidemic Preparedness and Response (EPR).

\section{Competing interest}

The authors declare that there are no competing interests. The publication of this article was approved by the dean School of Pure and Applied Sciences, Kenyatta University.

Acknowledgement: We are also grateful to the study participants from Kamasai, both adults and children together with their parents/guardians. We also appreciate the effort of support staff from the local community, Kenyatta University and the Director KEMRI.

\section{REFERENCES}

1. World Health Organization. World Health Organization (WHO) 2015 malaria updates. World Health Organ Tech Rep Ser. 2015

2. Schwartz L, Brown GV, Genton B, Moorthy VS. A review of malaria vaccine clinical projects based on the WHO Rainbow table. Malar J. 2012;9(11): 11 .

3. Pierce SK, Miller LH. World Malaria Day 2009: What Malaria Knows about the Immune System That Immunologists Still Do Not. J Immunol. 2009;182:5171-5177.

4. Ojakaa N, Ofware PJ, Machira WRJ, et al. Community perceptions of malaria and vaccines in the South coast and Busia regions of Kenya. Malar J. 2011;10: 147.

5. Division of Malaria Control. 2010, Kenya Malaria indicator Survey. Division of Malaria Control, Kenya Ministry of Public Health and Sanitation Report. 2011.

6. Brooker S, Clarke S, Njagi JK, et al. Spatial clustering of.malaria and associated risk factors during an epidemic in a highland area of western Kenya. Trop Med Int Health. 2004;9(7):757-766.

7. John CC, Aaron JT, Moormann AM, Sumba PO, David EL, Xinan MM. Antibodies to pre-erythrocytic Plasmodium falciparum antigens andrisk of clinical malaria in Kenyan children. J Infect Dis Med. 2008; 197: 519-526.

8. Crompton PD, Pierce SK, Miller LH. Advances and challenges in malaria vaccine development. J Clin Invest. 2010;120(12): 114-118.

9. Marsh K, Kinyanjui S. Immune effector mechanisms in malaria. Parasite Immunol. 2006;28(1-2):51-60.

10. Coppi A, Natarajan R, Gabriele PG, Bennett BL, James ER, Roggero MA. The malaria circumsporozoite protein has two functional domains, each with distinct roles as sporozoites journey from mosquito to mammalian host. World J Exp Med. 2011;208(2): 341-356.

11. World Health Organization World malaria report. World Health Organ Tech Rep Ser. 2012.

12. Hoffman SL, Goh LML, Luke T.C, et al. Protection of humans against malaria by immunization with radiation attenuated Plasmodium falciparum sporozoites. J Infect Dis Med.2002;185:1155-1164.

13. Hillier CJ, Ware LA, Barbosa A, Angov E, Lyon JA, Heppner DG. Process development and analysis of liver-stage antigen 1, a pre- 
erythrocyte-stage protein-based vaccine for Plasmodium falciparum. Infection Immun. 2005;73(4): 2109-2115.

14. Mccall MBB, Sauerwein RW. Interferon: A central mediator of protective immune responses against the pre-erythrocytic and blood stage of malaria. J Leukoc Biol. 2010;88(6): 1131-1133.

15. Fontaine A, Pophillat M, Bourdon S, Villard C, Belghazi M, Fourquet, P. Specific antibody responses against membrane proteins of erythrocytes infected by Plasmodium falciparum of individuals briefly exposed to malaria. Malar J. 2010;9: 276.

16. Jun SO, Jang SK, Chang HL, Deok HN, Sun HK, Dae WP. Evaluation of a malaria antibody enzyme immunoassay for use in blood screening. Memorias do Instituto Oswaldo Cruz. 2008;103(1):75-78.

17. Khan ZM, Vanderberg JK. Specific inflammatory cell infiltration of hepatic schizonts in BALB/C mice immunized with attenuated Plasmodium yoelii sporozoites. Int $\mathrm{J}$ Immunol Immunother. 1992;4(7):711-718.

18. Some ES. Effects and control of highland Malaria in Uasin Gishu district Kenya. East Afr Med J. 1994;71(1):2-8.

19. Sarr JB, Remoue F, Samb B. Evaluation of antibody response to Plasmodium falciparum in children according to exposure of Anopheles gambiae s.l or Anopheles funestus vectors. Malar J. 2007;6:117.

20. John CC, McHugh MM, Moormann AM, Sumba PO Ofulla AV. Low prevalence of Plasmodium falciparum infection among asymptomatic individuals in a highland area of Kenya. Trans. R. Soc. Trop. Med. Hyg. 2005;99:780-786.

21. Noland GS, Paterson BH, Min XM, et al. Low Prevalence of Antibodies to Preerythrocytic but Not Blood-Stage Plasmodium falciparum Antigens in an Area of Unstable Malaria Transmission Compared to Prevalence in an Area of Stable Malaria Transmission. J Infection Immun. 2008; 76(12):5721-5728.

22. Ofulla AV, Moormann AM, Embury PE, Kazura JW, Sumba PO, John CC. Age-related differences in the detection of Plasmodium falciparum infection by PCR and microscopy, in an area of Kenya with holoendemic malaria. Annu Trop Med and Parasitol. 2005;99:431-435.

23. Frevert U, Sinnis P, Cerami C, Shreffler W, Takacs B, Nussenzweig V. Malaria circumsporozoite protein binds to heparin sulfate proteoglycans associated with the surface membrane of hepatocytes. J Exp Med. 1993;177: 1287-1298.

24. Chatterjee S, Wery M, Sharma P, Chauhan VS. A conserved peptide sequence of the Plasmodium falciparum circumsporozoite protein and antipeptide antibodies inhibit Plasmodium berghei sporozoite invasion of Hep-G2 cells and protect immunized mice against P. berghei sporozoite challenge. Infect and Immun. (1995). 63: 4375-4381.

25. Sultan AA. Molecular mechanism of malaria sporozoite motility and invasion of host cells. Int J Microbiol Curr Res. 1999;2:155-160.

26. Muller HM, Reckmann I, Hollingdale MR, Bujard H, Robson KJ, Crisanti A. Thrombospondin related anonymous protein (TRAP) of Plasmodium falciparum binds specifically to sulfated glycoconjugates and to HepG2 hepatoma cells suggesting a role for this molecule in sporozoite invasion of hepatocytes. EMBO J. 1993;12:2881-2889.
27. Hay SI, Noor AM, Simba M, et al. Clinical epidemiology of malaria in the highlands of western Kenya. J Emerg Infect Dis. 2002;8:543-548.

28. Snow RW, Omumbo JA, Lowe B, et al. Relation between severe malaria morbidity in children and level of Plasmodium falciparum transmission in Africa. Lancet 1997;349:1650-165.

29. Riley, E. M.; Allen, S. J.; Wheeler, J. G.; Blackman, M. J.; Bennett, S.; Takacs, B.; Schonfeld, H. J.; Holder, A. A. and Greenwood, B. M. (1992). Naturally acquired cellular and humoral immune responses to the major merozoite surface antigen (PfMSP1) of Plasmodium falciparum are associated with reduced malaria morbidity. Parasite Immunol. 14:321-337.

30. Scarselli E, Tolle R, Koita O, et al. Analysis of the human antibody response to thrombospondin-related anonymous protein of Plasmodium falciparum. Infection Immun. 1993;61: 3490-3495.

31. Roussilhon C, Oeuvray C, Müller-Graf C, Tall A, Rogier C, Trape JE. Long-term clinical protection from falciparum malaria is strongly associated with IgG3 antibodies to merozoite surface protein 3. PLoS Med. 2007;4(11):320.

32. Osier FH, Fegan G, Polley SD, et al. Breadth and magnitude of antibody responses to multiple Plasmodium falciparum merozoite antigens are associated with protection from clinical malaria. Infection Immun. 2008;76:2240-2248.

33. Tongren JE, Drakeley CJ, McDonald SL, et al. Target antigen, age, and duration of antigen exposure independently regulate immunoglobulin $\mathrm{G}$ subclass switching in malaria. Infection Immun. 2006;74:257-264.

34. World Health Organization. World malariareport 2013. World Health Organ Tech Rep Ser. 2013.

35. World Health Organization. Q\&A on Malaria Vaccine Implementation Programme (MVIP). World Health Organisation. Geneva, Switzerland. 2019.

Peer Reviewed

Competing Interests: None declared.

Received: 13 Jun 2019; Accepted: 09 May 2020.

Cite this article as: Walukhu MK, Gicheru MM, Nyukuri RW. Low exposure to Plasmodium falciparum and Acquisition of Antibodies to Circumsporozoite Protein Antigens in Individuals Living in Western Highlands of Kenya with Unstable Malaria Transmission. E Afr Sci. 2020;1(2):66-75. http://doi.org/10.24248/EASci-D-19-00017

(C) Walukhu et al. This is an open-access article distributed under the terms of the Creative Commons Attribution License, which permits unrestricted use, distribution, and reproduction in any medium, provided the original author and source are proper-ly cited. To view a copy of the license, visit http://creativecommons.org/licens- es/by/4.0/. When linking to this article, please use the following permanent link: http://doi.org/10.24248/EASci-D-19-00017 